\title{
EL DISCURSO REPRODUCIDO EN DISCURSOS DE POSESIÓN PRESIDENCIAL SUDAMERICANOS*
}

\author{
Finiza Polanía-Beltrán \\ Ruprecht-Karls-Universität Heidelberg \\ fpolaniabeltran@gmail.com
}

Resumen: Basado en el modelo de Koch y Oesterreicher (2007), que defiende la tesis de que el lenguaje oral y el escrito presentan unas características particulares que no solamente se limitan a su forma, este trabajo analizará el discurso político como un corpus escrito. Siendo los discursos de Alberto Fernández, Jeanine Añez, Sebastián Piñera, Iván Duque, Lenin Moreno, Martín Vizcarra y Nicolás Maduro los discursos de interés. Así pues, el objetivo de este trabajo es analizar el uso del discurso reproducido en discursos de posesión presidencial sudamericanos. En este sentido, se buscará comprender las causas y las motivaciones detrás de ello así como su uso. El trabajo de Concepción Maldonado (1991) sobre el discurso reproducido es fundamental para esta tarea.

Palabras clave: Discurso reproducido, verba dicendi, discurso directo, discurso indirecto, discursos presidenciales.

Title: Reproduced speech in South American presidential inauguration addresses

\begin{abstract}
Based on the model of Koch and Oesterreicher (2007), which defends the thesis that oral and written language present particular characteristics that are not only limited to their form, this paper will analyze political discourse as a written corpus. The speeches of Alberto Fernández, Jeanine Añez, Sebastián Piñera, Iván Duque, Lenin Moreno, Martín Vizcarra and Nicolás Maduro are the speeches of interest. Thus, the objective of this paper is to analyze the use of the discourse reproduced in South American presidential inauguration addresses. In this sense, it will seek to understand the causes and motivations behind it as well as its use. Concepción Maldonado's (1991) work on reproduced discourse is fundamental to this task.

Keywords: Reproduced speech, verba dicen$d i$, direct speech, indirect speech, presidential addresses.
\end{abstract}

"Este trabajo ha sido realizado en el marco del seminario «Español hablado, Español escrito», del Romanisches Seminar de la Universidad de Heidelberg, guiado por la Dr. Lena Sowada, a quien agradezco sus valiosos consejos y sugerencias. 


\section{INTRODUCCIÓN}

Quienes no son lingüistas deben tomarse unos minutos para responder si un discurso, de aquellos que se declaman ante un público, pertenece a la oralidad o a la escrituralidad. Pues aunque su ejecución suele ser desde la inmediatez verbal, su concepción ha sido desde lo escrito; es decir, desde la distancia comunicativa. Koch y Oesterreicher (2007) sugieren que el idioma y sus variedades, el género, la forma o el estilo, moldean la manera como el lenguaje es utilizado y deben tenerse en cuenta en el momento de analizar el discurso, aquel que alude al acto comunicativo. Junto a estos debe añadirse también la concepción del mismo: oral o escrita, la primera caracterizada por la inmediatez y la segunda por la distancia comunicativa. Sin embargo, algunos casos suelen resultar particulares por su naturaleza dual, como una entrevista en una publicación, hecha de manera oral para ser divulgada de manera escrita, o una obra de teatro, cuyos diálogos fueron adaptados del papel. Este es el caso del discurso público, aunque se transmite de manera oral no suele gestarse frente a nosotros, sino que ha tenido una planificación escrita, especialmente cuando las alocuciones provienen de figuras públicas. El discurso, particularmente el discurso político, nuestro objeto de estudio aquí, que en ocasiones ha sido aprendido de memoria y puede llegar a añadir improvisaciones para dar la impresión de espontaneidad, normalmente ha sido planeado con anterioridad y generalmente suele ser leído, por lo que no puede ser considerado como parte de la inmediatez comunicativa. Precisamente el corpus analizado aquí será considerado como un documento escrito, pues partimos de su concepción, aspectos referentes a la oralidad como la prosodia o la entonación serán excluidos, no sin reconocer la complejidad de hacerlo y su fuerte presencia en este objeto de estudio, como se descubrirá más adelante.

El objetivo de este trabajo es analizar el discurso reproducido dentro del discurso presidencial. La fuente principal de las reflexiones aquí presentadas es el trabajo de Concepción Maldonado, Discurso directo y discurso indirecto (1991), quien junto a otros, nos ayudará a profundizar en la noción del discurso reproducido, sus características y clasificaciones. El corpus agrupa cada uno de los primeros discursos ofrecidos por los presidentes procedentes de siete países sudamericanos: Argentina, Bolivia, Chile, Colombia, Ecuador, Perú y Venezuela. Los presidentes respectivamente son: Alberto Fernández, Jeanine Añez, Sebastián Piñera, Iván Duque, Lenin Moreno, Martín Vizcarra y Nicolás Maduro․ Durante todo este trabajo, las citas utilizadas para ejemplificar serán extraídas directamente del corpus mencionado, los

1 Este trabajo fue llevado a cabo durante los primeros meses de 2020. A día de hoy, la situación política de los países aquí presentados ha cambiado y varios de sus mandatarios también. 
discursos de posesión presidencial. El objeto de estudio, es decir, el discurso político, será presentado y delimitado para así continuar con su análisis y la identificación del fenómeno lingüístico de nuestro interés: el discurso reproducido. Este análisis será realizado en diferentes categorías: el quién, el por qué y el cómo; las cuales facilitaran una comprensión más amplia del discurso reproducido. En último lugar, serán presentadas algunas consideraciones finales.

\section{EL DISCURSO REPRODUCIDO}

Es aquel que le permite al enunciador representar, reconstruir o reproducir declaraciones de otra situación discursiva. Para Coulmas (1986: 2) es el lenguaje para referir al lenguaje, el habla sobre el habla, enunciados como sujetos de otros enunciados. Este es llamado también discurso citado, ajeno, o más comúnmente referido. Este último, indica Maldonado acertadamente, «tan sólo describe una acción realizada verbalmente», mientras que el término discurso reproducido «reproduce esa situación de enunciación. Reproducir supone siempre referir, pero no al contrario» (1991: 20). De ahí la inclinación de este trabajo por la noción discurso reproducido (DR), pues es además una noción bien establecida en la lengua española. El uso del DR no es solamente una estrategia «universal del lenguaje» (Maldonado, 1991: 11), sino que además, afirma Carrizo (2012), es una estrategia de naturaleza funcional, comunicacional, interaccional y persuasiva.

Funcional porque se establece una relación muy estrecha entre el marco discursivo y los recursos lingüísticos usados. Comunicacional porque los distintos tipos de DR actúan sobre el destinatario, funcionan conativa y, por rebote, tácticamente: la acción verifica el contacto. Interaccional porque, al implicar un diálogo, el receptor que responde se ve interpelado a desplazarse en el rol discursivo que le toca: pasa a ser auditorio activo del intercambio. Por último, es una estrategia persuasiva porque organiza el contenido proposicional, de acuerdo con una estructura argumental en juego, con el propósito de evitar el rechazo de la posición que se defiende. (Carrizo, 2012: 125)

Precisamente el énfasis en el contenido proposicional al que se refiere Carrizo, qué dijo el enunciador en lugar de cómo lo dijo, incide en que se pierdan «los elementos emocionales y afectivos y la reformulación [se realice] de acuerdo con los nuevos propósitos y con el nuevo texto que se construye» (Nigro y Tonelli, 2013: 39). Por ejemplo, (1) corresponde al enunciado original y (1a) corresponde al enun- 
ciado citado; mientras que en el primero el hablante menciona dos caminos opuestos que podrían suceder en el país, con cierto tono de incertidumbre, en el segundo enunciado el hablante refiere la cita de forma imperativa, con un tono que parece irrefutable.

(1) O seguimos paralizados en nuestro desarrollo [...] o [...] nos lanzamos, con decisión y coraje a la conquista del futuro por el camino del progreso y de la grandeza del país. (Frondizi, 1958)

(1a) Como alguna vez dijera Arturo Frondizi, debemos lanzarnos «con decisión y coraje a la conquista del futuro». (Fernández, 2019)

Así que el verbo utilizado para reproducir un discurso no será necesariamente el mismo verbo del enunciado original ni un sinónimo, «sino que calificará de otro modo el acto de habla de la fuente» (Nigro y Tonelli, 2013: 39), ya que la reproducción del discurso depende de cómo el hablante que refiere interprete lo que se dijo o como quiera que su audiencia lo interprete.

\subsection{Verba dicendi o verbos de decir}

No cualquier tipo de verbo, sin embargo, indica un discurso reproducido. Maldonado (1991: 35) afirma que para que una cita sea efectivamente reproducida el enunciado que la introduce debe incluir un verbo de comunicación verbal flexionado, conocidos también como verba dicendi o verbos de decir, ambas definiciones serán utilizadas de manera indistinta en las páginas siguientes. Estos «pueden definirse como los que remiten a un acto de decir» (Nigro y Torelli, 2013: 40), o como Reyes (1995: 16-17) señala, «expresan las actividades verbales de los seres humanos, pero no todas, sino las que son específicamente comunicativas, es decir, las que tienen la intención prioritaria de transmitir algo, una información, a otra persona ». Irónicamente, señalan Nigro y Torelli (2013: 40), todos los verbos de decir, menos decir, aportan información sobre el acto lingüístico efectuado. Por ejemplo, al comparar los verbos reclamar y decir puede verse que el primero indica una exigencia, en (2), mientras que el segundo, en (3), no insinúa nada en particular, «sólo anuncia un discurso» (Reyes, 1995: 17).

(2) Los casados sabemos que lo primero que nos reclaman las esposas es: «¿cuándo compramos una casita?» (Moreno)

(3) Lao Tse decía que para estar delante del pueblo uno debe ir siempre detrás del pueblo. (Moreno) 
El grupo de los verba dicendi es abundante. Brunetti (2009: 78) los agrupa en declarativos, de opinión, de valoración, de manera de decir, de orden, de petición y los que lexicalizan la modalidad de la enunciación, como son los verbos que indican la verdad, los que sitúan el discurso citado en la orientación argumentativa, los que explicitan la fuerza ilocutiva del discurso citado, los que inscriben el discurso reproducido en una tipología de las distintas formas de narrar, o los que especifican el modo de realización fónica del enunciado. Por eso Maldonado (1991: 36) señala que en el idioma español los verbos de comunicación verbal, a diferencia de los verbos de percepción sensible, de pensamiento o de sentimiento, se comportan de distinta manera, pues según ella estos indican que las citas son reproducción de palabras, bien sean propias o ajenas, reales o hipotéticas, pronunciadas o escritas. Sin embargo, Brunetti (2009:79) reconoce que «muchos verbos son usados como verbos de comunicación aun cuando no pertenezcan a este campo». En la sección 4, de análisis y discusión de resultados, será retomado nuevamente este tema y ahondaremos en esta divergencia.

\subsection{Condiciones del discurso reproducido}

No solamente el uso de un verbo de comunicación verbal garantiza que estamos frente a un caso de DR. Maldonado (1991: 17) expone los que deberían ser requisitos o condiciones que un enunciado lingüístico debe cumplir para que pueda ser reconocido como discurso reproducido: metarreferencialidad, representatividad y no realizatividad.

\subsubsection{Condición de la metarreferencialidad}

Cuando el objeto de una situación de enunciación, señala Maldonado (1991: 17), sea otra situación de enunciación. Además, incluye situaciones discursivas sencillas, como en (4), hasta situaciones discursivas más complejas de enunciaciones atribuibles a múltiples fuentes (Maldonado, 1991: 17). En (5), el hablante cita al presidente del máximo tribunal de la república, quien a su vez ha citado un artículo de la constitución.

(4) El poeta viajero, Walt Whitman, decía: lo que yo tengo, lo tienes tú... cada átomo de mi cuerpo, es tuyo también. (Moreno)

(5) Y este artículo, como bien lo explicaba el presidente del máximo tribunal de la República, [...] Este artículo 231 es taxativo -así dicen, ¿̇verdad?-, taxativo y dice, oído, atención artículo 231: «El candidato elegido o candidata elegida 
tomará posesión del cargo de Presidente o Presidenta de la República el 10 de enero del primer año de su período constitucional, mediante juramento ante la Asamblea Nacional — punto y seguido. Porque para los abogados no es lo mismo el punto y seguido que el punto y aparte. Punto y seguido. Quiere decir que es continuidad del concepto-. Si por cualquier motivo sobrevenido el Presidente o Presidenta de la República no pudiese tomar posesión ante la Asamblea Nacional, lo hará ante el Tribunal Supremo de Justicia». (Maduro)

Aunque en situaciones de múltiples fuentes se suele «preferir el uso del discurso indirecto por la complejidad que supone hacer uso de varios sistemas de referencias deícticas distintos, especialmente en el lenguaje oral» (Maldonado, 1991: 17), (5) es un caso particular, pues está en DD, principalmente porque lo citado es un artículo de la constitución nacional y se presume que su exactitud es importante para su credibilidad.

\subsubsection{Condición de la representatividad}

Un enunciado no puede considerarse reproducido si solamente describe un hecho comunicativo realizado, según Maldonado decir: «igritó!» o «Cantó», solamente describe las características físicas de un sonido y « es necesario que la cadena verbal en la que se hace referencia al enunciado ajeno, esté representado de algún modo [el objeto del discurso] y que no se trate sólo de la mención de un acto lingüístico» (Maldonado, 1991: 20). En (6) observamos el verbo cantar en una situación de discurso reproducido pues en efecto encontramos un objeto del discurso, un enunciado referido.

(6) Como ese entrañable bolero de Farrés, cantado por Los Panchos: «Toda una vida». Desde el mismo momento de la concepción hasta cuando Dios decide cerrarnos los ojos. (Moreno)

\subsubsection{Condición de la no realizatividad}

Los enunciados pueden ser reproducidos, explica Maldonado (1991: 29), a través de verbos de significado locutivo (decir, comunicar, etc.) o ilocutivo (preguntar, avisar, ordenar etc.), pero no deben estar usados con un valor realizativo; «es decir, si no aparecen en presente y en primera persona (con esas características, el mero uso de estos enunciados no supone la descripción de una acción sino su realización)» (Maldonado, 1991: 29). Diferente al uso del presente histórico, como expone Galluci (2009: 83), donde se emplea el tiempo verbal presente en la narración de hechos pasados, que retomaremos en breve. 
Entonces para que un enunciado sea considerado reproducido, debe reproducir «una situación verbal introducida por verbos de decir usado de forma descriptiva, no realizativa, y que la situación de enunciación reproductora tenga como objeto la situación de enunciación reproducida» (Maldonado, 1991: 29). Por ejemplo, mientras que el enunciado expresado por Martín Vizcarra: «Estoy dispuesto a asumir la responsabilidad que marca nuestra Constitución Nacional», es un enunciado realizativo, pues la mención a la constitución solo enfatiza una acción que se pretende realizar, en (7), Alberto Fernández menciona la constitución y además describe una de sus cláusulas, convirtiéndose así en un enunciado reproducido.

(7) Reafirmamos nuestro más firme compromiso con el cumplimiento de la Cláusula Transitoria 1ra de la Constitución Nacional y trabajaremos incansablemente para potenciar «...el legítimo e imprescriptible reclamo por la soberanía sobre lasIslas Malvinas, Georgias del Sur y Sandwich del Sur y los espacios marítimos e insulares correspondientes...». (Fernández)

Como se dijo anteriormente, existen «contextos en los que el presente morfológico no coincide con el presente real del momento de enunciación» (Maldonado, 1991: 28), como en el caso del presente histórico. Por ejemplo, en (8) tenemos la recreación de una enunciación en tiempo pasado, en donde podemos ver cómo se mezcla el uso del presente. Sin embargo, Maldonado también afirma que este tipo de construcciones no son exclusivas del presente histórico, sino también de «aquellos enunciados en los que la marca aspectual concede a la oración el valor de habitualidad» (1991: 28). Como por ejemplo, (9) y (10), con el adverbio de tiempo siempre, indicando habitualidad, pero el verbo conjugado en presente. Por otro lado, la enunciación (11) usa el mismo adverbio, pero la conjugación del verbo está en tiempo pasado.

(8) Una periodista del Washington Post creo que era - Jorge- Rachel Siegel del Washington Post mepreguntaba $;$ Es verdad que usted el 10 de enero empieza su dictadura? Yo le digo, pónganse de acuerdo, ¿cuándo empezó, ustedes no decían que Chávez era el dictador? (Maduro)

(9) Yo le digo a nuestro pueblo siempre: «allá ellos con su desesperación, su nerviosismo y su locura» (Maduro)

(10) Yo siempre le digo a los invitados internacionales vayan por las calles, hablen con la gente donde quiera, párense en la plaza que quieran y hablen con el pueblo. (Maduro)

(11) Siempre he dicho y lo seguiré diciendo, que aquí lo que esperan los colombianos son soluciones y no agresiones. (Duque) ${ }^{2}$

2 Cursivas añadidas en los cuatro enunciados para enfatizar los verbos. 
Todos estos son ejemplos que nos permiten observar las distintas herramientas comunicativas del discurso reproducido y reafirmar la compleja naturaleza.

\subsection{Tipos de discurso reproducido}

Convencionalmente se identifican dos tipos de discurso reproducido: el discurso directo (DD) o el discurso indirecto (DI). Sin embargo, afirma Coulmas (1986: 6), las complejidades del discurso representado no se pueden hacer justicia a través de esta simple dicotomía, por lo que existen además otros procedimientos en lengua castellana para reproducir un discurso. Entre los cuales encontramos el discurso indirecto libre, el pseudo-indirecto, el indirecto mimético, el directo libre, el directo no regido o el pseudo-directo. Estos tipos de discurso reproducido están más presentes en el lenguaje escrito, y aunque inicialmente se aclaró que el corpus analizado se tomará como texto escrito, estos procedimientos se manifiestan mayormente en narraciones literarias o textos periodísticos, mientras que la particularidad del discurso político limita la posibilidad de análisis de estos, por lo que nuestro enfoque aquí serán el DI y el DD. Según Coulmas (1986: 2), su diferencia radica en la perspectiva o punto de vista de quien reporta; aunque también se dice que depende de «la fidelidad relativa de lo citado a lo originalmente proferido por las voces invocadas» (Prieto y San Martín, 2002: 283).

\subsubsection{El discurso directo}

Para Coulmas (1986: 2), el DD reproduce una situación discursiva y transmite, o intenta transmitir, las palabras exactas del hablante original. Maldonado (1991:16) señala que estas palabras pueden ser propias o ajenas; es decir que cuando un hablante reproduce lo que él mismo ha dicho también estamos hablando de DR. Cuando las palabras reproducidas no son las propias, el hablante está de alguna manera modificando su punto de vista, pues como Coulmas (1986:2) señala, en el discurso directo, el reportero presta su voz al hablante original y dice (o escribe) lo que dijo, adoptando así su punto de vista. Coulmas (1986: 2) enfatiza además que el DD, no es el discurso del reportero, sino que sigue siendo el discurso del hablante reportado cuyo papel es desempeñado por el reportero. El uso de la cita directa (CD) en (12), permite que la voz de la fuente, Miguel Grau, suene en el mismo nivel que la del hablante, Martín Vizcarra, y al mismo tiempo que conserven su independencia, como una polifonía, tal como sugiere Carrizo al describir el DD como un «despliegue de una doble enunciación configurada polifónicamente a través de dos locutores» (2012: 175). Además, en (12) y en (13) podemos observar que en 
los enunciados en discurso directo la cita directa puede ubicarse, respectivamente, después o antes del verbo.

(12) Nuestro héroe Miguel Grau, en una de sus frases más importantes, dijo: «Yo no soy más que un pobre marino que trata de servir a su Patria ». (Vizcarra)

(13) «Tal vez de la suma o la confrontación de esas verdades podamos alcanzar una verdad superadora », supo decir con acierto Néstor Kirchner. (Fernández)

Sabemos entonces que el DD necesita al menos una CD. Pero, además de una $\mathrm{CD}$, Maldonado afirma que un DD debe estar constituido por una expresión introductoria (EI) la cual tiene un verbo de decir flexionado. Estas «están separadas por una pausa, marcada tipográficamente por los dos puntos» (Maldonado, 1991: 29), como en (12), y una coma, como en (13). Justamente en las marcas que señala Maldonado debemos establecer una expansión en esta ecuación, pues no todas las CD están siempre marcadas tipográficamente por guiones o comillas, ni separadas por dos puntos. En la oralidad no se puede tener esto en cuenta, ni tampoco en la escritura donde existe mucha libertad en la grafía, particularmente en el corpus que será analizado aquí. Por ejemplo, si en (14), agregáramos las marcas tipográficas correspondientes (14a), tendríamos la estructura sintáctica perfecta para categorizarla dentro del DD.

(14) y alguna gente del mundo que nos visita se preguntará, ¿’por qué Venezuela, Colombia y Ecuador tienen la misma bandera hermosa? (Maduro)

(14a)y alguna gente del mundo que nos visita se preguntará: «¿por qué Venezuela, Colombia y Ecuador tienen la misma bandera hermosa?» (Maduro)

Siendo nuestro objeto de estudio de una naturaleza tan especial, cuya complejidad se sugirió en el inicio y será discutida a profundidad más adelante, la decisión de considerar un enunciado DD no estará limitada a estas marcas, sino también a la independencia situacional establecida «con respecto al eje deíctico del hablante» (Payá, 2003: 13), pues como explica Payá, «la cita directa supone la creación de una situación comunicativa autónoma, en principio desligada del eje narrador, en la que $[\ldots]$ no son necesarias las variaciones deícticas» (2003: 13).

\subsection{El discurso indirecto}

El DI adapta las palabras reportadas, propias o ajenas, «desde el sistema de referencias deícticas del hablante que reproduce» (Maldonado, 1991: 16). Este permite una mayor subjetividad al reproducir el discurso. Aquí, afirma Coulmas (1986: 2), el 
hablante relaciona el evento discursivo desde su punto de vista, existiendo una dependencia situacional entre la cita y el verbo declarativo en el nivel sintáctico, como explica Payá (2003: 14), «el texto citado, introducido generalmente por la conjunción que, se subordina» al verbo «como complemento de objeto directo del mismo».

Todo discurso indirecto (DI) está constituido por una expresión introductora (EI) que contiene un verbo de decir flexionado, una cita indirecta (CI) cuya marca es la conjunción que, y el contenido citado (CC). La CI está subordinada al verbo de la EI. (Maldonado, 1991: 30)

En (15) y (16) tenemos el uso de la conjunción que y la dependencia a la que Payá se refiere, lo que provoca una especie de fusión entre los discursos; en (17) el hablante recrea sus propias palabras, siendo un enunciado «autocitado» (Galluci, 2009: 80).

(15) Yo estoy convencido, como lo dijo en alguna ocasión Federico García Lorca cuando fue a inaugurar la biblioteca de su pueblo, Fuente Vaqueros, en España, que hay que entregar un pan y un libro, porque no hay peor hambre que el hambre de conocimiento, que no se puede saciar. (Duque)

(16) Stephen Hawking me dijo alguna vez que ya es hora de cambiar de concepción de existencia; que hasta ahora hemos vivido con la mecánica clásica de Newton y que es hora de empezar a pensar ya con los nuevos conceptos científicos físicos de la relatividad y la mecánica cuántica; que hay que empezar a pensar en aquellas cosas que van más allá de la velocidad de la luz y que en más de una ocasión nos desconciertan, por aquello que parece inaccesible. (Moreno)

(17) Le prometí a Colombia que como Presidente asumiría esa tarea con entusiasmo. (Duque)

\section{EL DisCURSO POLÍtico COMO OBJETO DE ESTUdIO}

Para Charaudeau (2002: 116) el discurso político es aquel que busca justificar, legitimar y dotar de credibilidad a quienes poseen y ejercen el poder. De Arnoux (2021: 733) sostiene que este surge, y se inscribe, «en el marco de actividades sociales destinadas a alcanzar, gestionar o defender el poder» sostenido además «por locutores legitimados institucionalmente y que responde a los temas de la agenda pública». Los locutores a los nos referimos aquí son los presidentes de la nación, quienes se comunican por distintas razones y de distintas maneras con el pueblo que gobiernan: alocuciones presidenciales televisadas, comunicados, ruedas de prensa, y en los últimos años también a través de publicaciones en las cuentas oficiales en sus redes sociales. Aunque 
no existe una regularidad en la frecuencia y medio de las comunicaciones, todos ofrecen un discurso oficial el día de su investidura, también conocido como discurso de posesión, inaugural o de asunción. Este primer discurso suele coincidir en menciones a las propuestas durante la campaña, al plan de gobierno, alusiones al gobierno anterior y a los problemas más importantes que enfrenta el país. Pero debido a su característica optimista y utópica al sugerir el inicio de nueva era con el comienzo de sus gobiernos, podría considerarse como lo que Charaudeau (2002: 113) ha llamado «discurso de seducción y de persuasión», pues justamente la finalidad de este tipo de discursos «construir imágenes y efectos, más que ideas», creando al mismo tiempo un imaginario de pertenencia comunitaria.

El discurso político como un tipo de interacción social, según Villarraga (2012: 37), crea entonces grupos ideológicos y por eso recibe gran atención en los estudios sobre análisis crítico del discurso. Sin embargo, Charaudeau (2002: 123) afirma que «sería ingenuo pensar que [el objeto del análisis dcl discurso político] sería únicamente el contenido ideológico del discurso, a menos que se redefina la ideología», pues el análisis del discurso político se relaciona y se ve influenciado por diferentes componentes como los hechos políticos, sociales, jurídicos, morales y psíquicos. Es por todo esto que Fairclough (2013: 73) afirma que el discurso está conformado por estructuras, pero también contribuye a moldearlas y remodelarlas, a reproducirlas y transformarlas.

Sin embargo, Armony (2005) señala que la validez del análisis del discurso presidencial se ve cuestionada cuando se piensa en que el presidente no suele redactar sus alocuciones oficiales o no lo hace solo, y que estas están dadas de tal forma que lo beneficien, resultando oportunistas, con una función puramente retórica; «el discurso es visto entonces como un encadenamiento de fórmulas vacías, convenciones y lugares comunes» (Armony, 2005: 34). No obstante, continúa Armony, quién analiza el discurso no tiene la responsabilidad justiciera de un periodista, y justamente son estos aspectos los que resultan atractivos: el poder simbólico, la legitimidad de la institución estatal, los ajustes y realineamientos del discurso, las constantes, los puntos de densidad, lo permitido o no, los «clichés» propios al discurso político, la «mismidad»; es decir,

(el carácter de lo que permanece igual a sí mismo y coincide exactamente con su representación). El Estado asume un papel particular en la producción de un discurso fundador de lo «mismo» totalizador y unificador la Nación, el Pueblo, el Destino que borra la historicidad inmanente y restablece una ilusión de trascendencia (la Nación es siempre igual a sí misma; la Nación y su idea son ontológicamente lo mismo). Las palabras mágicas, las palabras sagradas, las palabras que «hacen creer» (Bourdieu, 1982), todas esas palabras que nombran una «plenitud comunitaria ausente» (Laclau, 2000) son las palabras 
que se repiten. Las palabras (y las asociaciones de palabras) recurrentes son las huellas de una fijación parcial de la significación social y cumplen, por ello, un papel clave en la reproducción del orden social. (Armony, 2005: 37)

Lo que señala Armony también argumenta la decisión de considerar el discurso presidencial como corpus escrito ${ }^{3}$, pues las palabras han sido preparadas y buscan cumplir con patrones de discursos ya existentes; sin embargo, cada vez más los políticos buscan imprimir un estilo personal, como será visto en estas páginas.

\subsection{Datos relativos al corpus}

El corpus está constituido por siete discursos políticos sudamericanos, procedentes de Argentina, Bolivia, Chile, Colombia, Ecuador, Perú y Venezuela. Particularmente nos referimos al primer discurso ofrecido, ver cuadro 1. Los presidentes respectivamente son: Alberto Fernández, Jeanine Añez, Sebastián Piñera, Iván Duque, Lenin Moreno, Martín Vizcarra y Nicolás Maduro. Todos los discursos son públicos, de fácil acceso, y las transcripciones fueron tomadas de sitios oficiales estatales de cada país (acceso a los discursos completos en las fuentes primarias de la bibliografía).

\begin{tabular}{|c|c|c|c|c|c|c|c|}
\hline Presidente & Añez & Duque & Fernández & Maduro & Moreno & Piñera & Vizcarra \\
\hline $\begin{array}{c}\text { Número } \\
\text { de palabras }\end{array}$ & 695 & 5.172 & 7.216 & 7.887 & 6.863 & 3.018 & 1.363 \\
\hline Fecha de emisión & 15.11 .19 & 7.08 .18 & 10.12 .19 & 10.01 .19 & 24.03 .17 & 11.03 .18 & 23.03 .18 \\
\hline $\begin{array}{c}\text { Número de } \\
\text { enunciados } \\
\text { referidos } \\
\text { encontrados }\end{array}$ & 0 & 10 & 9 & 15 & 21 & 4 & 1 \\
\hline
\end{tabular}

Cuadro 1. Información general de los discursos

La situación política de América Latina es, a lo menos, agitada; tres de los presidentes aquí mencionados están rodeados de situaciones inverosímiles. Martín

\footnotetext{
Como fue mencionado inicialmente, la ya bien conocida propuesta de Koch y Oesterreicher, y su modelo sobre los aspectos concepcionalmente relevantes de la oralidad y escrituralidad, permite establecer una diferencia entre el lenguaje oral y escrito, justificando también la consideración del discurso político como corpus escrito. Ya que el discurso político cuenta con las condiciones y estrategias propias de la distancia comunicativa: es público, con poca emocionalidad y familiaridad, sucede en la distancia física, sin carácter dialógico, permite la reflexión y posee una fijación temática. Además, se caracteriza por su planificación y carácter definitivo (Koch y Oesterreicher, 2007: 34).
} 
Vizcarra se posesionó luego de que el presidente electo Pedro Pablo Kuczynski renunciara el día anterior acusado de corrupción; Nicolás Maduro no es reconocido como presidente por varios países, mientras coexiste con la figura internacional de Juan Guaidó; Jeanine Añez, asumió el cargo temporalmente luego de la renuncia de Evo Morales acusado de fraude electoral, mientras se convocan a nuevas elecciones. A diferencia de los demás, Añez no promulgo oficialmente un discurso de toma presidencial, así que el primer discurso publicado en una página oficial después de asumir el cargo es el que ha sido utilizado.

\section{ANÁLISIS Y DISCUSIÓN DE LOS RESULTADOS}

Siendo el corpus analizado de carácter político resulta inevitable eludir algunos aspectos relevantes, pues así como señala Kranert (2019:9), se reconoce el deseo de los políticos de influir en las acciones de la gente generando un cambio opinión en ellos, siendo la retórica una herramienta de persuasión importante. Por esto el análisis de nuestro corpus se ha dividido en tres categorías, considerado los siguientes aspectos: las personas que se citan, con qué fines y cómo lo hacen.

\section{1. ¿A quién citan?}

Los discursos citados provienen de fuentes variadas, desde ex presidentes y poetas hasta amigos y familiares. Identificando características en común, se han formado subcategorías: célebre, propio, familiar, material, ficticio, popular, desconocido. Un total de 61 situaciones de discurso reproducido fueron encontradas, ver gráfica 1 . El $50 \%$ pertenece al denominado discurso célebre, discursos de personajes reconocidos y que los hablantes de alguna manera admiran.

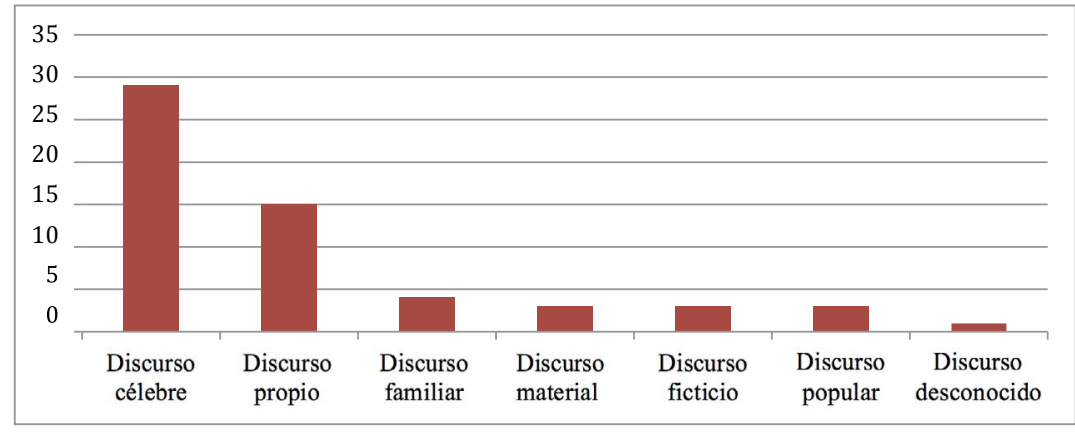

Gráfica 1. Clasificación de los discursos 
Dentro del discurso celebre, Lenin Moreno destaca por el excesivo uso que le da. Los personajes utilizados son muy variados, desde políticos y militares hasta escritores y religiosos, ver gráfica 2 ; sin embargo, los enunciados emitidos por políticos son los que más se recrean. Contrario a conjeturas iniciales sobre una abundancia de referencias al discurso de los héroes de la independencia, solamente se encontraron dos enunciados, (18) y (19). Sin embargo, esto no significa que no se haga referencia a los considerados héroes de la patria, bien sea, personajes notables, políticos o militares. Por ejemplo, existe una clara elección por citar ex presidentes, como se ve en (1a), (13), (20), (39), (49) y (58). Parece ser que estos son preferidos pues resultan más actuales y son más recordados por la audiencia.

(18) No se equivocó Simón Bolívar cuando anunciaba: «La unidad de nuestros pueblos no es simple quimera de los hombres, sino inexorable decreto del destino». (Moreno)

(19) Como diría nuestro Padre de la Patria Francisco de Paula Santander, «un país que sabe honrar a sus héroes, es un país que sabe forjar su futuro». (Duque)

(20) En alguna ocasión el célebre Darío Echandía se preguntó: ¿El poder para qué? (Duque)

Dentro de este grupo se encontraron referencias a escritores y músicos, incluyendo poetas, ensayistas, cantautores. No se encontró predilección especial por la nacionalidad, algunas veces son personajes de gran reconocimiento internacional, como en (22) y (23), otras son personajes locales, como en (21) donde Moreno hace referencia a un escritor ecuatoriano no muy conocido.

(21) Juan Montalvo decía: «No soy enemigo de individuos ni de clases sociales. Donde está la corrupción, allí está mi enemigo». (Moreno)

(22) Octavio Paz, ese gran pensador y Premio Nobel mexicano, cuando hablaba del Estado, siempre se refería al «Ogro Filantrópico». Filantrópico, porque se supone que el Estado ayuda a la gente; y ogro, porque para muchos ciudadanos el Estado es alguien distante, lejano y hostil. (Piñera)

(23) Y estimular ese otro nacimiento, aquel que Savater llama el «nacimiento social», el del pensamiento simbólico, el de las palabras. (Moreno)

También se hace mención a conceptos o ideas originales expresados por intelectuales, (22) y (23), en donde la noción original conserva las marcas tipográficas mientras que la explicación del mismo se resume o parafrasea. 


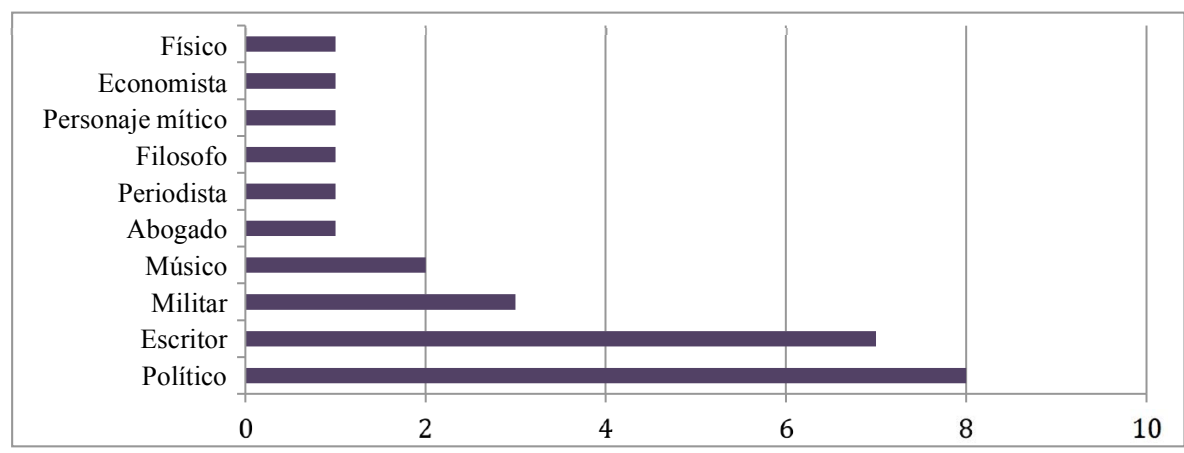

Gráfica 2. Tipos de personajes encontrados

Según Galluci (2009: 80), el discurso reproducido puede ser «autocitado» (del $y o$ ) o «heterocitado» (del otro); aunque la tendencia se inclina más hacia el segundo, encontramos en nuestra muestra un $25,9 \%$ de «autocitaciones», que aquí hemos categorizado como discursos propios. Estos representan enunciaciones hechas por los hablantes anteriormente y promesas hechas durante la campaña. En estas no siempre se habla en singular, (24), (25) y (26) lo muestran, lo que puede hacer referencia al hablante y a la audiencia, y también al hablante y su equipo de campaña, pues como se dijo anteriormente las declaraciones presidenciales suelen requerir asesores y un equipo que controla la imagen que se quiere transmitir.

(24) El futuro no espera. Lo dijimos: el futuro es ahora. (Moreno)

(25) Vamos a promover los pliegos tipo -como lo dijimos en campaña- en todos los sectores del Estado y vamos a limitar a no más de tres períodos la presencia en cuerpos colegiados de elección popular. (Duque)

(26) Quiero reiterar ante ustedes el compromiso que hicimos durante la campaña: las prioridades de nuestro gobierno van a ser las prioridades de los chilenos.(Piñera)

(27) pero llévense la seguridad hermanos del mundo como se los he dicho a ustedes en las conversaciones que hemos tenido, [...], la Revolución Bolivariana sabrá enfrentar estas amenazas, estas agresiones y así como en nuestros 20 años de Revolución sabremos remontar los obstáculos, las dificultades, y saldremos airosos, victoriosos, con mayor conciencia, con mayor compromiso (Maduro)

(28) Muchas veces me he preguntado en estos días por qué motivos quisiera que nuestro Gobierno sea recordado en el futuro (Fernández)

(29) Durante la campaña $-y$ antes de ella - dije que tenía mi mano extendida para todos. (Moreno) 
El discurso familiar hace referencia a personas que pertenecen al círculo cercano de los hablantes, amigos o familiares, lo que representa un $6,9 \%$ total, y cuyo contenido suele ser bastante más informal, como (30) y (31) lo exponen.

(30) Como dijo una vez una compañera. «Es contigo, María», «es contigo, Pedro», es contigo, compatriota, es con nosotros. (Maduro)

(31) Si la esposa dice «no me pasa nada »... retírate un poquito. Pero si vuelve a decir «no me pasa nada $» . .$. iHuye! (Moreno)

El discurso material, ficticio y popular se presentan en la misma cantidad cada uno, un 5,2 \%. El discurso material no hace referencia a enunciados pronunciados por personas, sino a enunciados provenientes de otro tipo de elementos, como clausulas legales o artículos de la constitución. Por ejemplo, Moreno cita el lema de la convención de las Naciones Unidas sobre los derechos de las personas con discapacidad (32), Maduro cita la constitución (5) y (36), Duque cita el lema del escudo de Colombia (33), Fernández cita una cláusula de la constitución (7).

(32) Esa lucha enarboló el lema «Nada sobre nosotros, sin nosotros». (Moreno)

(33) Esa legalidad construida por el matrimonio de seguridad y justicia nos garantizará el balance que se encuentra en el Escudo de Colombia y dice: LIBERTAD Y ORDEN. (Duque)

Cuando se habla del discurso ficticio se hace referencia a discursos de los que no se tiene la certeza si realmente sucedieron, pero los hablantes imaginan que quizás sí o que podrían suceder.

(34) A veces preguntamos a un político, «perdóneme, pero usted ayer opinaba lo contrario». (Moreno)

(35) Y si preguntan por cada uno de nosotros, y si ustedes preguntaran quién es Maduro, y yo mismo me preguntara quién soy yo. (Maduro)

Como puede observarse en (35), algunas citas no tienen una forma sintáctica clara. En (36), por ejemplo, no es posible comprender exactamente a quién se cita, pues se menciona a la constitución, al doctor Escarrá y al doctor Maikel Moreno. Siendo una marca irrefutable de la oralidad, pues la planeación de la escrituralidad permite evitar este tipo de dificultades de compresión.

(36) la Constitución de la República Bolivariana de Venezuela dejó establecido desde el año 99 que cada 10 de enero del primer año del mandato presidencial, una 
vez siendo electo el Presidente o la Presidenta tenía y tiene la obligación de presentar juramento ante la Asamblea Nacional, el Poder Legislativo, y establece de manera sabia, como bien lo ha comentado el doctor Escarrá recientemente en varios foros públicos, establece una excepción muy clara para cualquier causa sobrevenida pueda darse el acto de juramentación ante las autoridades del máximo Tribunal de Justicia, como bien lo certificó el presidente del Tribunal Supremo de Justicia, el doctor Maikel Moreno. (Maduro)

El discurso popular hace referencia a frases o refranes conocidos dentro del folclor cultural, aquellos que se repiten dentro de la sociedad, pero generalmente no se conoce su origen real. En (37), Moreno repite dos enunciados de este tipo, ambos con una intención semántica similar, por lo que se ha considerado todo como un solo enunciado. Y finalmente, con una enunciación, el discurso desconocido es aquel, como su nombre lo indica, donde la identidad de la fuente desconocemos, pues no se menciona, como en (38). Sin embargo, el que no se identifique la fuente no indica necesariamente que el orador la desconozca. Podría indicarnos, como sugiere Escribano (2008: 217), que en este caso el valor de la enunciación no radica en el prestigio de su autor sino en el contenido proferido.

(37) Y vamos escuchando por ahí proverbios, refranes que dicen «Árbol que nace torcido, jamás su tronco endereza»o «Genio y figura, hasta la sepultura». (Moreno)

(38) Alguien decía que ya es hora de dejar atrás las ideologías. (Moreno)

En la siguiente sección se profundizará en las posibles razones detrás de estas referencias.

\section{2. ¿Por qué citan?}

El discurso político, según Kranert (2019: 30), tiene varias capas que nos permiten interpretar su intención semántica: el género, el léxico, la argumentación y la metáfora; entendemos aquí el uso del discurso reproducido como parte de la argumentación. Igualmente para Escribano (2008: 214) este recurso es «un poderoso mecanismo argumentativo» pues permite «esconder bajo el discurso propio otras voces que permiten apoyar la propia visión o criticar la del contrario, muchas veces de forma implícita y no evidente». Referirse a personajes célebres, figuras elevadas e incluso consideradas autoridades en su campo, explica Kray (2016: 424), podría servir para impresionar a la audiencia, mostrar erudición, afirmar competencia, infundir confianza o defenderse de las críticas. De hecho, señala Escribano (2008: 
216) «para que la cita sea capaz de intensificar el valor argumentativo del propio discurso, ha de ser de alguien con un prestigio indudable y además de reconocimiento público». Al citar, ex presidentes o políticos admirados por su buena gestión, es probable que los presidentes busquen que su audiencia establezca una conexión entre ellos y lo que será su gobierno, pues como explica Kray (2016: 424) su reputación se suma a la credibilidad del orador. Pero, además, como sugiere Escribano (2008: 216), el orador busca que las características intelectuales e ideológicas del personaje mencionado sean similares. Notorio, por ejemplo, en Fernández, quien cita principalmente a expresidentes argentinos con ideologías parecidas a las que él quiere poner en práctica durante su gobierno:

(39) En 1974, el general Juan Domingo Perón señalaba que «a niveles nacionales, nadie puede realizarse en un país que no se realiza. De la misma manera, a nivel continental, ningún país podrá realizarse en un continente que no se realice». (Fernández)

Como señalamos anteriormente, es probable que la reproducción del discurso de héroes independentistas no esté muy presente pues el uso excesivo que se ha hecho de él a lo largo de los años, como sugiere Kray (2016: 425), pueda resultar contraproducente o considerado como una mala utilización, por lo que prefieran utilizar «héroes» más actuales para evitar restarle fuerza a su argumento con lo que podría considerarse cliché. Igualmente el citar artistas, músicos, escritores muestra de cierta manera un intento para ostentar una erudición y una sensibilidad especial, situación que sucede principalmente en el discurso de Moreno, como (40) y (41) lo muestran. Como afirma Charaudeau (2009: 288), «hay argumentos que remiten al ethos del orador» y sirven así como «elemento de identificación para el auditorio», y es quizás por esto que se citan enunciados cuyos hablantes no representan ninguna autoridad, como familiares y amigos, tal como en (42), o enunciados de origen popular. Posiblemente porque estos enunciados también son una manera de dar información sobre sí mismos, mostrarse como personas accesibles, familiares y confiables, afines a la audiencia.

(40) Somos una sociedad que con inusitada frecuencia «archiva» a los ancianos después de que nos han servido bien. Así lo dice Joan Manuel Serrat. (Moreno)

(41) Mario Benedetti decía que el secreto para una relación duradera es el diálogo entre las diferencias. (Moreno)

(42) Días atrás un amigo me señalaba la importancia de todo ello en el futuro que se avecina. (Fernández) 
Por otro lado, la referencia a elementos como el escudo, cláusulas, leyes o artículos de la constitución no solo legitima, sino que de alguna manera, como sugiere Kray (2016: 426), orienta su discurso, pues los presidentes quieren incluirse dentro del marco de la ley, como es el caso de Nicolás Maduro, en (36) y en (5), cuyo gobierno y elección ha sido bastante señalado y rechazado. Quizás por eso se refieren a enunciados dichos por ellos mismos, como sugiere Butler (2009: 25), quizás lo hacen como forma para asumir la responsabilidad por los propios actos a través de este recurso. Aunque Butler no se refiere de forma directa al discurso político o a su rol, es posible establecer una relación sobre la forma en que nuestros hablantes «dan cuenta de sí» y la afirmación de que «damos cuenta de nosotros mismos únicamente porque se nos interpela en cuanto seres a quienes un sistema de justicia y castigo ha puesto en la obligación de rendir cuentas» (Butler, 2009: 22). Al recrear enunciados de la campaña, los presidentes están reafirmando la obligación que adquirieron a través de las promesas hechas y de sus propias afirmaciones, no es una rendición de cuentas como tal, pues su gobierno no ha comenzado, pero sí es una reafirmación frente a esa promesa.

(43) ¡arriba los corazones, porque llegó el momento de esos tiempos mejores, de los cuales hablábamos durante la campaña, para todos ustedes y para todas las familias chilenas! (Piñera)

(44) Yo lo dije ayer, el mundo es más grande que el imperio estadounidense y sus gobiernos satélites (Maduro)

Los presidentes al hacer referencia a sus propios enunciados, establecen una especie de lema propio, apelando a su propia voz y autoridad, reafirmando su poder. Finalmente como afirma Kranert (2019: 9), el lenguaje político es una herramienta de persuasión pues, como dice Charaudeau (2009: 283), el actor político busca persuadir al mayor número de individuos para reafirmar su legitimidad.

\section{3. ¿Cómo citan?}

Como fue discutido anteriormente, solo los verbos de comunicación verbal nos indican una situación de discurso representado. En total se identificaron 65 verbos de comunicación verbal en los discursos, que no corresponde necesariamente al número de enunciados reportados, pues algunas enunciaciones utilizaron dos verbos. En (45), se ve como el referir una conversación hace necesario el uso dos verbos, pues hay una pregunta y respuesta; aunque a veces también podemos encontrar la repetición del mismo verbo, como en (46). 
(45) Quisiera recordarles la frase del economista John Maynard Keynes —allá por los tiempos del New Deal de Franklin Delano Roosevelt - cuando un periodista le preguntó: Perdóneme señor Keynes pero usted hace un año pensaba diferente. Y él le contestó: Sí señor, porque cuando yo me equivoco cambio de opinión. ¿Qué es lo que hace usted? (Moreno)

(46) El Padre Hurtado, ese gran Santo chileno, decía que la Patria es mucho más que nuestras cordilleras, nuestros valles o nuestro mar. Decía que la Patria era una misión a cumplir. (Piñera)

Sin embargo, puede observarse que la reformulación de la frase también puede resultar en que se modifique el verbo inicial, para cambiar el sentido, como (47) y (48).

(47) Y aquí tomo unas frases que algún momento encontré en el Kymalión, este libro de dialéctica escrito supuestamente por Hermes Trimegisto - hace ocho mil años, dicen - aquel que luego fuera convertido y prostituido como «Mercurio». La corrupción, dice Hermes Trimegisto, parafraseo lo que él dijo en verdad: es como un cuarto lleno de oscuridad. Para que esta acabe, no hay que sacar la obscuridad, hay que introducirle luz, llenarle de luz. (Moreno)

(48) Yo diría, como en varias oportunidades yo he afirmado, Venezuela tiene adversarios muy poderosos y dañinos (Maduro)

La recreación de situaciones discursivas largas, como una anécdota, también genera un desafío para su clasificación. Como en (49), en donde se incluyen diferentes verbos, aunque reconocemos como verbo introductorio el verbo decir.

(49) $\mathrm{Al}$ respecto hay una anécdota del general Carlos Soublette, dos veces presidente de Venezuela. Soublette conoció que había una obra de teatro en la cual se lo ridiculizaba. Llamó al autor de la obra y le dijo:

- Mire señor, conozco que usted está haciendo una obra de teatro ama «Excelentísimo Señor» (no sé si «ese lentísimo» o «es el lentísimo») y que se ríe de mí.

- $\quad$ Sí señor Presidente, le dijo el autor de la obra a Soublette.

- Hijo, ¿pudiera verla?

- Encantado, señor Presidente.

Presentaron la obra y en ella Soublette se rió mucho. No la objetó ni la vetó, ante el asombro del autor, que dijo:

- Señor Presidente, ¿podemos presentar esta obra en la cual la gente se ríe de usted?

Soublette le contestó:

- La República no se perderá porque el pueblo se ría de un gobernante; se perderá cuando el gobernante se ría de su pueblo. (Moreno) 
En la gráfica 3 puede observarse la preferencia del uso de los verbos; aunque puede afirmarse que las gráficas en este segmento son concluyentes, los resultados de alguna manera no son totalmente exactos, pues como se ha visto existen situaciones que deben ser consideradas de forma independiente y algunos enunciados merecen ser discutidos individualmente y por esto han sido excluidos de las gráficas por falta de certeza. Sin embargo, es evidente la inclinación de los hablantes hacia el uso del verbo decir para reproducir un discurso, con más de la mitad de las enunciaciones (38 en total).

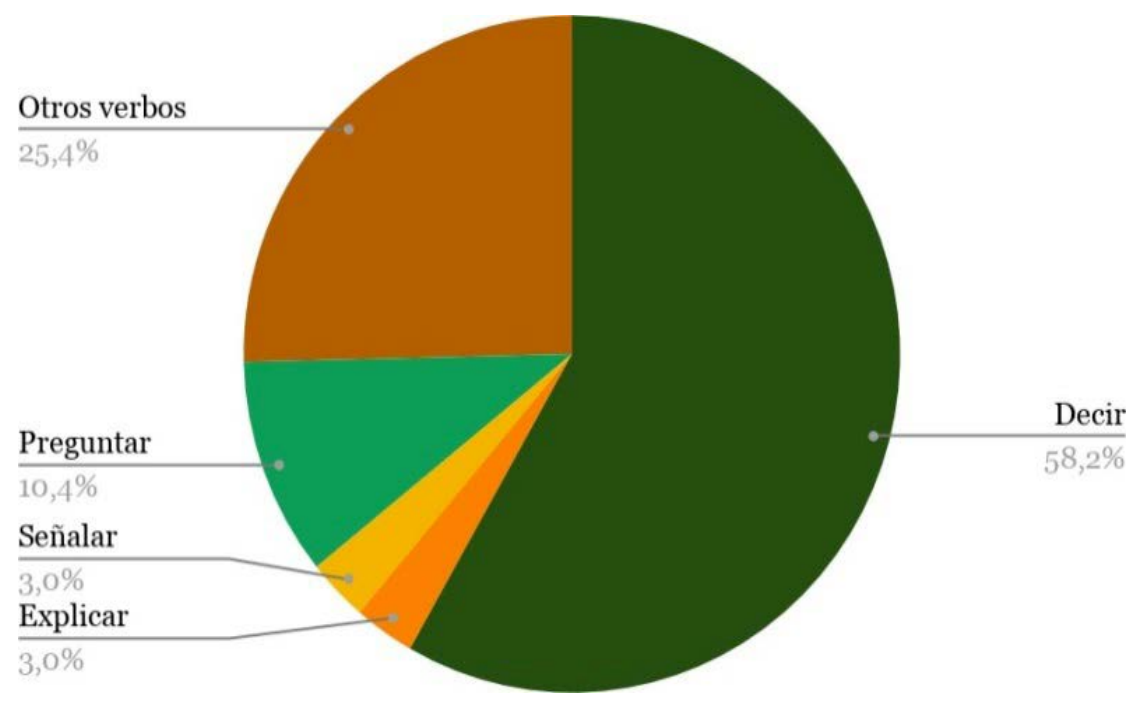

Gráfica 3. Verbos utilizados

El siguiente verbo más utilizado es preguntar que solo representa un 10,4\% de uso (un total de 7 enunciaciones), seguido de explicar y señalar ambos con dos enunciaciones cada uno, 3,0 \%, y el 25,4\% restante, un total de 17 verbos, cada uno con una sola enunciación.

\subsubsection{El verbo decir}

Como fue mencionado anteriormente, a excepción de los demás verbos de comunicación verbal que aportan información sobre el acto lingüístico, decir 
no lo hace. Por esto suele ser considerado como un verbo neutral; sin embargo, la predilección de su uso para reportar el discurso se debe no solamente a su valor transitivo, afirma Payá(2015:423), sino a que posee ciertos rasgos que lo equiparan a verbos intransitivos como hablar ya que establece «una doble posibilidad, a saber, la de usar y mencionar el lenguaje simultáneamente». Además, podría resultar también una «forma estratégica que tiene el hablante de no comprometerse con el tipo de afirmación que efectuó en su día el hablante citado» (Payá, 2003: 18), pues cualquier otro verba dicendi lo haría de formadiferente. En toda la muestra fueron encontradas distintas maneras en el uso del verbo decir, en diferentes posiciones dentro del enunciado: antes de la cita (50), en el interior (51) y después de la cita (52). En diferentes tiempos verbales, ver gráfica 4 , siendo el uso del indicativo (Ind.) ampliamente preferido sobre el subjuntivo.

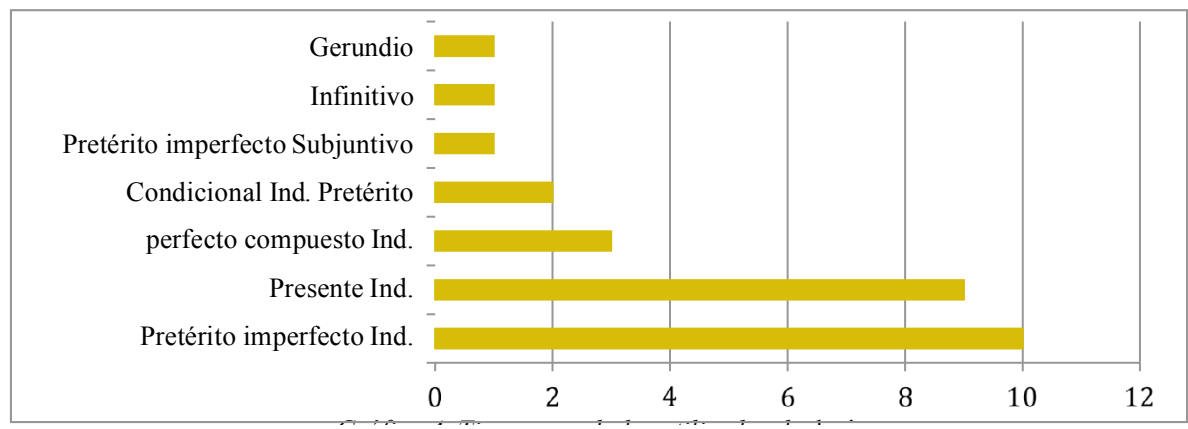

Gráfica 4. Tiempos verbales utilizados de decir

Puede verse que sobresale el uso del tiempo pasado en formas sencillas, pretérito perfecto simple y pretérito imperfecto. Lo que muestra que los hablantes buscan la mayor claridad y simplicidad en la estructura de su discurso. Aunque resulta interesante el uso del infinitivo del verbo decir con el Pretérito perfecto simple del verbo saber, en (53), hemos contado el verbo de comunicación verbal al verbo decir.

(50) Yo he dicho que no pretendo ser un presidente encumbrado y encerrado en un palacio (Duque)

(51) Aprender a aprender — decía Lenin — para mejor comprender y mejor actuar. (Moreno)

(52) Para todo ello, repito con Monseñor Leonidas Proaño: ¡Bienvenidos luchadores de la paz y de la vida! ¡Bienvenidos luchadores de la paz y de la vida, decía Monseñor Proaño! (Moreno) 
(53) Supo decir un penalista clásico, que cuando la política ingresa a los Tribunales, la justicia escapa por la ventana. (Fernández)

Igualmente, la conjugación del verbo varía no solamente en tiempo, sino también en persona. Como en los tres casos ejemplificados abajo, todos se encuentran en pretérito perfecto simple del indicativo, pero en diferentes personas, primera y tercera persona del singular y primera persona del plural respectivamente.

(54) yo lo dije: llueva, truena o relampaguee, Venezuela va a seguir por el camino de la democracia y la paz (Maduro)

(55) Como dijo el profesor Luis Brito García en un foro también, echaron de lado los conceptos fundamentales, básicos, históricos de la humanidad relacionados a la soberanía, a la autodeterminación de los pueblos y actúan de manera grosera, extravagante contra nuestro país (Maduro)

(56) durante la campaña dijimos que tendríamos un gabinete de personas expertas en sus materias, que no respondieran a cálculos políticos sino a su vocación de servicio y a sus resultados (Duque)

Todo esto nos permite decir que en general para reproducir un discurso existen múltiples formas, no obstante, las estructuras más sencillas y de fácil comprensión suelen ser más utilizadas pues facilitan la transmisión de la idea que se quiere expresar.

\subsubsection{Otros verbos}

Entre los verba dicendi encontrados en el objeto de estudio, hay un 41,8\% de verbos diferentes al verbo decir, estos son: preguntar, señalar, explicar, reafirmar, prometer, comentar, afirmar, anunciar, parafrasear, contestar, repetir, referir, llamar, reclamar, certificar, cantar, hablar, reiterar, enarbolar. Un 25,4\% de ellos solo con una enunciación. Entre ellos algunos presentan una polisemia semántica, pero el contexto y el contenido de cita que se reproduce es lo que permite identificar el sentido correcto. Igualmente, existen otros verbos que presentan un desafío mayor, pues generalmente no serían considerados como verba dicendi, estos son hacer y tener. En (57), (58) y (59), puede verse como se utilizan estos verbos para referir el discurso, en todos los casos junto con una palabra que modifica de alguna manera su condición realizativa: hacer valer, tener razón y tener sentido.

(57) haciendo valer ese principio de «El que la hace, la paga». (Duque)

(58) Ese día quisiera poder demostrar que Raúl Alfonsín tenía razón. Espero que en- 
tre todos podamos demostrar que con la democracia se cura, se educa y se come. (Fernández)

(59) En la relación con los periodistas, más que nunca tiene sentido aquella frase de que «las cuentas claras conservan la amistad y el respeto». (Fernández)

No es extraño que muchos verbos que no son considerados como verbos de comunicación verbal sean utilizados como tales y aun así su significado sea fácilmente comprendido, pues como afirma Brunetti (2009: 80), para esto el contexto verbal tiene un rol fundamental, situación que puede verificarse en los ejemplos presentados anteriormente, pues de manera aislada no serían considerados verbos para referir el discurso.

\subsubsection{Procedimientos utilizados para referir un discurso}

En este apartado se discutirá sobre los tipos de DR que fueron delimitados anteriormente: el DD y el DI. Sin embargo, aquí no se logró un resultado tan concluyente pues aunque la concepción del discurso presidencial ha sido escrita, presenta rasgos fuertes de oralidad por su forma de ejecución. Esto es más perceptible, por ejemplo, en el discurso de Nicolás Maduro, cuyos enunciados llevan en muchos casos la marca típica de la oralidad. En (60), por ejemplo, la expresión, que ayer tuvo full sintonía ¿no?, nos remite inmediatamente a un lenguaje oral, al utilizar un anglicismo coloquial.

(60) como bien lo explicaba el compañero Diosdado Cabello anoche en su programa, que ayer tuvo full sintonía ¿no?, Con el mazo dando. Más o menos. (Maduro)

Ahora, la importancia de señalar esto radica en que la clasificación de los enunciados en DD o DI no resulta tan sencilla debido a la flexibilidad con las marcas tipográficas en donde textos escritos tradicionales las llevarían, ya que este descuido no resulta tan relevante para quien lee un discurso, pues hará las pausas y entonaciones correctas para que la audiencia las identifique. Sin embargo, como se dijo anteriormente, el análisis que haremos no puede estar limitado bajo estas marcas. El discurso de Maduro particularmente presenta más descuidos de este tipo; sin embargo, sí puede decirse que hay una inclinación por el uso de las estructuras sintácticas correspondientes al DD, ver gráfica 5 , un $52 \%$ contra un $43 \%$ de DI. Según Paya (2003: 18), esto quizás porque «el empleo del discurso directo [...] persigue el mismo objetivo que cualquier hecho comunicativo, esto es, convencer al otro de que lo que decimos es cierto», mientras que en el uso del DI, 
focaliza no tanto la producción exacta del mensaje como si el contenido del mismo; el hablante que se hace responsable de la reproducción de ese contenido básico supedita el espacio mental del conceptualizador al suyo propio. Por ello aunque existen dos espacios mentales (i.e. del hablante y del conceptualizador), en realidad ambos espacios se mezclan y, en último término, prevalece el espacio del hablante reproductor de la cita (Payá, 2003: 15)

No es difícil imaginar que aunque nuestros hablantes utilicen el DR como estrategia de argumentación y autoridad quieran establecer su propio discurso y sus propias ideas, y prefieran el uso del DD precisamente para establecer una diferenciación entre los espacios mentales que menciona Payá.

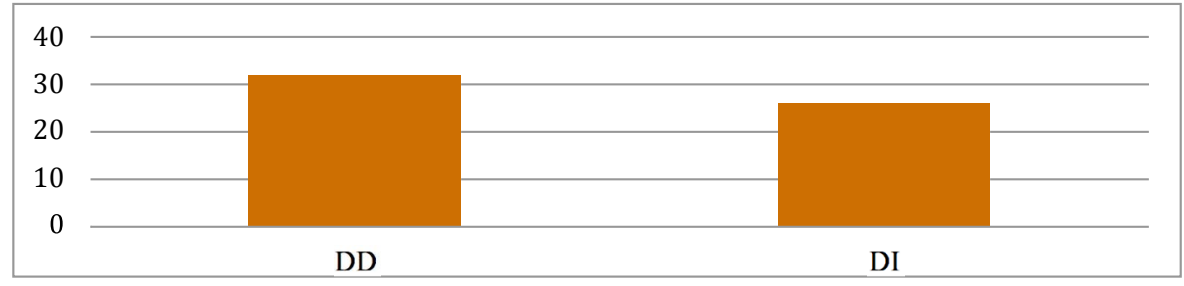

Gráfica 5. Tipos de discurso reproducido

Existen además otros casos en donde el enunciado referido pareciera estar en ambos modos, lo que Reyes (1984: 81) llama una cita mixta (cita directa dentro de la cita indirecta), como en (61) donde el primer enunciado se encuentra en DI y el segundo, lo que responde Maduro, está en DD. En este caso, podrían dividirse los enunciados y contarse separadamente, pero el sentido completo del enunciado radica en esta especie de dialogo; es decir, en la cita completa.

(61) años antes nos decían que nosotros exagerábamos cuando calificábamos de fascista a buena parte de la derecha opositora venezolana, y le decíamos: «No, lo que hay que tener cuidado es que esta derecha nos termine infectando y contaminando de intolerancia ideológica, de fascismo y de extremismo de derecha al resto de la derecha democrática que existía entonces en América Latina y el Caribe». (Maduro)

Igualmente en (22) y (23) está en comillas el concepto original, pero la explicación del mismo está hecha en DI, similar ocurre en (6). Por lo que estos enunciados no son fácilmente clasificables. En estos últimos casos, podría decirse que esta estrategia es utilizada para resumir contenidos que de otra manera serían muy largos. 


\section{Conclusiones}

Los discursos políticos resultan un objeto de estudio intrincado, no solo por la dificultad de su clasificación, pues cuando al tomarlos como muestra escrita, debido a su concepción, encontramos rasgos característicos de la oralidad, sino también por su característica persuasiva y su trasfondo ideológico, que nos obliga a juzgarlo con ojos más atentos. Evidentemente hay diferencias entre los hablantes analizados, pues como ha afirmado Kranert (2019: 15), el contexto global del discurso puede ser visto, en el sentido más amplio, como un contexto cultural, y debido a la diversidad existente en los contextos políticos y culturales de cada país, no sorprende la existente diversidad en los discursos que ofrecen los políticos de cada uno de estos. Así pues, el análisis de los discursos permite concluir que existen diferentes elementos que influyen en su elaboración, más allá del estilo personal que rige el estilo de los discursos, pues no todos nuestros hablantes tienen el mismo historial educativo, social y cultural. Por ejemplo, el tiempo de preparación del discurso está directamente relacionado con la complejidad y extensión del mismo, como podría ser el caso de los discursos de los presidentes de Bolivia y Perú, quienes debieron asumir el poder debido a la renuncia del presidente elegido, que son mucho más cortos que los demás, y escasamente utilizan el DR, solo una enunciación de Vizcarra y ninguna de Añez. Igualmente, es posible señalar que la relación que se tiene con el gobierno anterior también influye en la elaboración del discurso, pues en el caso de Moreno en el momento de ser elegido hacía parte del mismo partido político de su predecesor, así que su discurso tuvo un estilo menos alarmante y agresivo, mucho más optimista y vivaracho, lo que le permitió valerse de múltiples enunciaciones citadas, especialmente de figuras literarias.

El discurso reproducido, entendido como una herramienta argumentativa, permite descubrir diferentes intenciones persuasivas por parte de los hablantes que hemos analizado, de la imagen que quieren mostrar de sí mismos y las figuras e ideas con las que quieren ser asociados. Es por eso que figuras políticas reconocidas y con opiniones afines son las más referidas. Así pues, el uso del DR, a través de estructuras claras y sencillas, busca aportar credibilidad a los locutores y se convierte entonces en una herramienta para seducir a la audiencia. 


\section{Bibliografía}

\section{Fuentes primarias}

Casa Rosada presidencia Argentina (2019): «Palabras del presidente Alberto Fernández en su acto de asunción ante la Asamblea Legislativa». En línea: <https://www.casarosada.gob.ar/informacion/discursos/46596palabras-del-presidente- alberto-fernandez-en-su-acto-de-asuncionante-la-asamblea-legislativa, $>$ [19/02/2020].

Congreso República del Perú (2018): «Discurso del presidente constitucional de la república, Ingeniero Martín Alberto Vizcarra Cornejo, ante el Congreso nacional, el 23 de marzo de 2018». En línea: <http://www.congreso. gob.pe/Docs/participacion/museo/congreso/files/mensajes/2001-2020/ files/mensaje-2018-2.pdf>. [19/02/2020].

Embaja de Bolivia en Nicaragua (2019): «Discurso de la ministra de relaciones exteriores, en la posesión de los ministros de s.e. la presidenta constitucional del estado plurinacional de Bolivia Jeanine Añez Chávez». En línea: <https://embolnic.com/2019/11/15/discurso-de-la-ministrade-relaciones-exteriores-en-la- posesion-de-los-ministros-de-s-e-lapresidenta-constitucional-del-estado-plurinacional-de- bolivia-jeanineanez-chavez/,> [19/02/2020].

Fundación Frondizi (1958): «Discurso presidencial del 1. ${ }^{\circ}$ de mayo de 1958 ante la asamblea legislativa». En línea: <http://www.fundacionfrondizi.org. ar/docs/discursos/12_file.pdf> [19/02/2020].

Ministerio del Poder Popular para relaciones exteriores de Venezuela (2019): «Discurso de juramentación de Nicolás Maduro como presidente constitucional de la República Bolivariana de Venezuela para el período 2019-2025». En línea: <http://mppre.gob.ve/discurso/juramentaciontsj-nicolas-maduro-presidente/,> [19/02/2020].

Prensa presidencia gobierno de Chile (2018): «Chile somos todos y debemos soñarlo, dibujarlo y construirlo entre todos: el primer discurso del Presidente Piñera en el Palacio de La Moneda $>$. En línea: <https://prensa. presidencia.cl/comunicado.aspx $? \mathrm{id}=71719>[19 / 02 / 2020]$.

Presidencia República de Colombia (2018): «El Pacto por COLOMBIA / Discurso de Posesión del Presidente de la República, Iván Duque Márquez». En línea: <https://id.presidencia.gov.co/Paginas/prensa/2018/El-Pactopor-COLOMBIA-Discurso-de-Posesion-del-Presidente-de-la-RepublicaIvan-Duque Marquez.aspx,> [19/02/2020]. 
Presidencia República de Ecuador (2017): «Discurso de posesión del presidente Lenín Moreno Garcés ante la asamblea nacional en presencia de jefes de estado, delegaciones e invitados nacionales e internacionales». En línea: <https://www.presidencia.gob.ec/wp-content/uploads/ downloads/2017/06/2017.05.24-DISCURSO-POSESI\%C3\%93NANTE- LA-ASAMBLEA-NACIONAL.pdf> [19/02/2020].

\section{Fuentes secundarias}

Armony, Víctor (2005): «Aportes teórico-metodológicos para el estudio de la producción social de sentido a través del análisis del discurso presidencial», Revista Argentina de Sociología, 3(4), pp. 32-54.

Brunetti, Paulina (2009): El discurso referido: formas canónicas y no canónicas de citación en la prensa diaria: aspectos teóricos y didácticos, Córdoba, Comunic-Arte.

Butler, Judith (2009): Dar cuenta de si mismo. Violencia ética y responsabilidad, Buenos Aires, Amorrortu.

CArrizo, Alicia E. (2012): La argumentación interaccional: efectos del uso del discurso referido, Buenos Aires, Biblioteca universidad de Buenos Aires [Tesis de doctorado].

Charaudeau, Patrick (2002): «¿Para qué sirve analizar el discurso político?», DeSigniS, 2, pp. 109-124.

Charaudeau, Patrick (2009). La argumentación persuasiva. El ejemplo del discurso político. Haciendo discurso. Homenaje a Adriana Bolivar, pp. 277 295.

Coulmas, Florian (ed.) (1986): Direct and indirect speech. Berlín, Walter de Gruyter.

De Arnoux, Elvira Narvaja (2021): «36 Los discursos de la política $»$, en Óscar Loureda y Angela Schrott (eds.), Manual de lingüistica del hablar, Berlín, De Gruyter, pp. 733-748. https://doi.org/10.1515/9783110335224-037

EsCRIBANO, Asunción (2007): «El discurso reproducido en las informaciones electorales», Análisi: quaderns de comunicació $i$ cultura, 35, pp. 65-82. En línea: <https://www.raco.cat/index.php/Analisi/article/view/74255> [19/02/2020].

EsCribano, Asunción (2008): «Usos lingüísticos polifónicos en el discurso sobre el terrorismo de Zapatero y Rajoy», Espacios Públicos, 11 (23), pp. 213-225.

Fairclough, Norman (2013): Critical Discourse Analysis. The Critical Study of Language, Nueva York, Routledge. 
Gallucci, María José (2009): «Nos fuimos a la casa, y mi mamá: “¿Estaban lanzándose por la quebrada?”, “¡No, mamá!”, “iClaro que sí!”: estudio piloto de las citas conversacionales en el habla de Caracas», Núcleo, 21 (26), pp. 75-98.

Koch, Peter y Wulf Oesterreicher (2007): Lengua hablada en la Romania: español, francés, italiano, Madrid, Gredos.

Kranert, Michael (2019): Discourse and Political Culture. The language of the Third Way in Germany and the UK, Ámsterdam, John Benjamins.

KraY, Thorn-R. (2016): «On Name-Dropping: The Mechanisms Behind a Notorious Practice in Social Science and the Humanities», Argumentation, 30(4), pp. 423-441. https://doi.org/10.1007/s10503-015-9362-6

Maldonado, Concepción (1991): Discurso directo y discurso indirecto, Madrid, Taurus.

Nigro, Patricia y Victoria Tonelli (2013): «Los verbos de decir y valor argumentativo en noticias de dos diarios de distribución gratuita de la ciudad de Buenos Aires», Revista Ecos de la Comunicación, 6(6), pp. 37-49.

Payá Benavent, Elisa (2003): «¿Por qué contamos nuestras historias cotidianas en estilo directo?», en Nicole Delbecque (ed.), Aproximaciones cognoscitivofuncionales al español, Ámsterdam, Brill Rodopi, pp. 11-20. https://doi. org/10.1163/9789004334403_003

Payá Benavent, Elisa (2015): Decir y discurso directo en los relatos de la conversación coloquial, Valencia, Biblioteca Universidad de Valencia [Tesis de doctorado].

Prieto, Luis y Abelardo SAn Martín (2002): «Diferencias de género en el empleo del discurso referido: aproximación sociolingüística y pragmáticodiscursiva $\gg$, Boletín de Filologia, 39(1), pp. 269-303.

Reyes, Graciela (1984): Polifonía textual: la citación en el relato literario, Madrid, Gredos.

REYES, Graciela (1995): Los procedimientos de cita: estilo directo y estilo indirecto, Madrid, Arco libros.

Villarraga, Ligia Yamila (2012): «Análisis del discurso de posesión de Juan Manuel Santos: la ideología de la Unidad Nacional», Forma y Función, 25(1), pp. 35-51.

Fecha de recepción: 30 de noviembre de 2020 Fecha de aceptación: 26 de abril de 2021 